\title{
The relativistic beaming model for active galactic nuclei
}

\author{
J.H. $\operatorname{Fan}^{1,2}$, G.Z. Xie ${ }^{3}$ and S.L. Wen ${ }^{4}$ \\ 1 CCAST (World Laboratory), P.O. Box 8730, Beijing, 100080, China \\ 2 Astrophysical Center, Guangzhou Teachers' College, Guangzhou, China \\ 3 Yunnan Observatory, Chinese Academy of Sciences, Kunming, China \\ 4 Department of Physics, Hunan Normal University, Changsha, China
}

Received May 23; accepted September 27, 1995

\begin{abstract}
In this paper a list of 48 objects with superluminal motions is compiled, and some of their properties are discussed.
\end{abstract}

Key words: galaxies: jets-galaxies: active-quasars: general-BL Lacertae objects: general

\section{Introduction}

The relativistic jet model (Blandford et al. 1977; Marscher 1978; Blandford \& Rees 1978; Blandford \& Konigl 1979) has been proposed to describe the observed properties of variable extragalactic radio sources associated with the nuclei of galaxies, quasars and blazars. This hypothesis has found increasing observational support, the most striking being perhaps the measurements of superluminal velocities in many of the sources which could be resolved by VLBI (Porcas 1987; Ghisellini et al. 1993).

In this paper, we present a sample of objects with superluminal motion and discuss some of their properties associated with beaming model. 0.5 .

Through out the paper, $H_{0}=100 \mathrm{~km} / \mathrm{s} / \mathrm{Mpc}, q_{0}=$

\section{The sample of superluminal sources and some statistical results}

\subsection{Sample}

Columns 1 to 7 in Table 1 give the name, redshift, visual magnitude $\left(m_{\mathrm{v}}\right)$, radio flux $(5 \mathrm{GHz})$ in Jy. Superluminal velocity $\left(\beta_{\text {app }}\right)$, maximum optical polarization and references.

\subsection{Statistical results}

Based on a beaming model, the following relations hold:

$$
f_{\nu}^{\text {ob. }}=\delta_{\nu}^{3+\alpha} f_{\nu}^{\text {in. }}
$$

$\beta_{\text {app }}=\beta_{\text {in }} \cdot \sin \theta /\left(1-\beta_{\text {in }} \cdot \cos \theta\right)=\delta \cdot \sin \theta \cdot \beta_{\text {in. }} /\left(1-\beta_{\text {in. }}^{2}\right)^{0.5}$

Send offprint requests to: J.H. Fan where $f_{\nu}^{\text {ob. }}$ and $f_{\nu}^{\text {in. }}$ are, respectively, the observed and intrinsic (corrected) flux densities, $\delta_{\nu}$ is the Doppler factor, $\theta$ is the angle of the jet to the line of sight, $\alpha$ is the spectral index.

For a single group of objects, we should expect

$$
m^{\text {in. }}=5 \log z+c_{1}
$$

and

$$
f^{\text {in. }}=-2 \log z+c_{2}
$$

From which it follows:

$$
\begin{gathered}
m_{\mathrm{v}}^{\text {ob. }}-5 \log z=-2.5(3+\alpha) \log \delta_{0} \\
\log f_{\nu}^{\text {ob. }}+2 \log z=(3+\alpha) \log \delta_{\nu}
\end{gathered}
$$

In the caseof a very small angle, $\theta$, i.e. $\sin \theta \sim\left(1-\beta_{\text {in }}\right)^{-0.5}$, $\delta \sim \beta_{\text {app }}$ can be obtained, and we should expect (here $\alpha o$ $=1.0$ and $\alpha r=0.0$ are adopted)

$$
\begin{gathered}
m_{\mathrm{v}}^{\mathrm{ob}}-5 \log z=-10 k \log \beta_{\text {app }}+c_{1} \\
\log f^{\text {ob. }}+2 \log z=3 \log \beta_{\text {app }}+c_{2}
\end{gathered}
$$

for the optical magnitude and radio flux density respectively, where $k$ is related with $\delta_{0}$ and $\beta_{\text {app }}$ by

$$
\delta_{0} \sim \beta_{\mathrm{app}}^{k}
$$

When the linear regression method is used to fit the data, the expected results have been obtained

$$
\begin{aligned}
m_{\mathrm{v}}^{\mathrm{ob}}-5 \log z= & -(3.35 \pm 0.81) \log \beta_{\text {app }} \\
+ & 20.25 \pm 0.25(N=42) \\
\log f_{\mathrm{R}}^{\mathrm{ob} .}+2 \log z & =(2 . \pm 0.23) \log \beta_{\text {app }} \\
& -1.31 \pm 0.07(N=41)
\end{aligned}
$$

with correlation coefficients being -0.48 and 0.54 , respectively, at a confidence level greater than $99 \% . N$ is the number of the considered objects. 


\section{Discussions and conclusions}

Apparent superluminal motion is common among core dominated quasars (Cohen 1989), superluminal motion and blazar properties are often related (Impey 1987), and superluminal motion and core dominance parameter are also related (Ghisellini et al. 1993).

In this paper, 48 objects with superluminal motion are compiled. 23 of which are highly polarized (Popt. > $3 \%$ ), 14 of which have low polarization (Popt $<3 \%$ ), and 3 of which, $0615+820,0723+679$, and $1040+123$, are not polarized; 34 are core dominated, and 10 are lobedominated; two, $0415+379$ and $0710+439$, are galaxies, 11 , $0235+164,0454+844,0716+714,0735+178,0851+202$, $1101+384,1749+701,1803+784,1807+698,2007+777$, and $2200+420$, are BL Lac objects. 3 objects, $0153+744$, $0235+164$, and $1807+698$, are not included in some of our discussions, the correlations of $\log f_{\mathrm{r}}+2 \log z-$ $\log \beta_{\text {app }}, m^{\text {ob. }}-5 \log z-\log \beta_{\text {app }}, \log \beta_{\text {app }}-\log z$, and $\log R-\log \beta_{\text {app }}$ for instance. $0235+164$ and $0153+744$, are candidates for gravitational lensing (Kuhr \& Schmidt 1990; Surdej et al. 1992), their apparent superluminal motion may come from the effect of the gravitational lens. As to $1807+698$, its optical Doppler factor is $\delta_{o}=1.24$; after consideration of its Doppler factor it fits the Hubble relation very well (Xie et al. 1993; Fan et al. 1993); its radio Doppler factor calculated from SSC model is $\delta_{\mathrm{R}}=0.36$ (Madau et al. 1987). This means the Doppler factor of $1807+698$ is very small, but its average apparent superluminal velocity is very high, $\beta_{\text {app }}=7.7 \mathrm{c}$. Obviously, it does not obey the condition: $\delta \sim \beta_{\text {app }}$. As listed in Table 1 , many objects have several determinations of their superluminal velocities. The velocity used to analyse is the average velocity in these cases.

\subsection{Superluminal motion and beaming model}

For the observations, we find $\log f_{\mathrm{r}}=-(0.13 \pm$ $0.03) \log z+c, m=(2.27 \pm 0.16) \log z+c$ (see Figs. 1 and 2), which do not fit the Hubble relations: log $f_{\mathrm{r}}=-2 \log z+c, m=5 \log z+c$. This is usually explained as duo to the fact that the emissions are strongly enhanced by the Doppler effect (Blandford \& Konigl 1979; Worrall 1986; Fan et al. 1993). If so, we should expect that the corrected magnitude and radio flux density fit the Hubble relations well. The corrections can be expressed as Eqs. (7) and (8) in the case of small angles. From the data, the expected results have been obtained:

$$
\begin{gathered}
m_{\mathrm{v}}^{\mathrm{ob} .}-5 \log z=-(3.35 \pm 0.81) \log \beta_{\text {app }}+20.25 \pm 0.25, \\
\log f^{\text {ob. }}+2 \log z=(2 . \pm 0.23) \log \beta_{\text {app }}-1.31 \pm 0.07
\end{gathered}
$$

with correlation coefficients being -0.48 and 0.53 at a confidence level greater than $99 \%$ (see Figs. 3 and 4). Comparing Eq. (7) and the statistical result, one finds

$$
\delta_{0} \sim \beta_{\mathrm{app}}^{1 / 3},
$$

which means the enhancement of optical luminosity is associated with the superluminal motion.

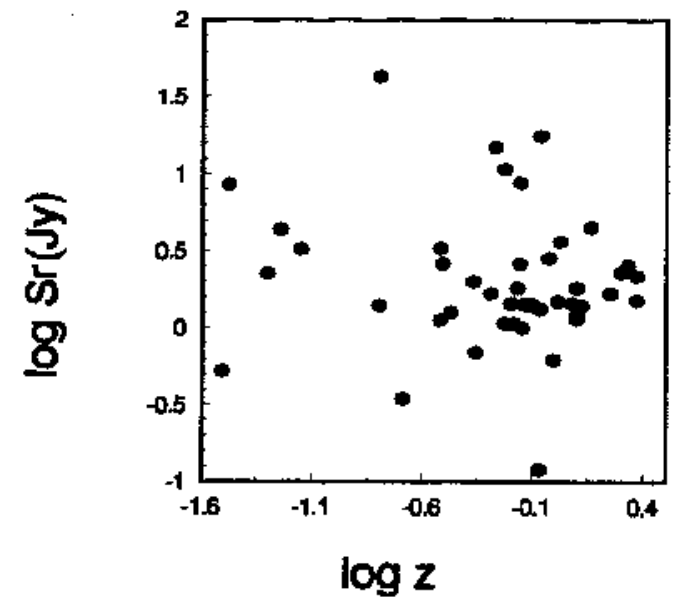

Fig. 1. Relation of radio flux density $(5 \mathrm{GHz})$ and redshift

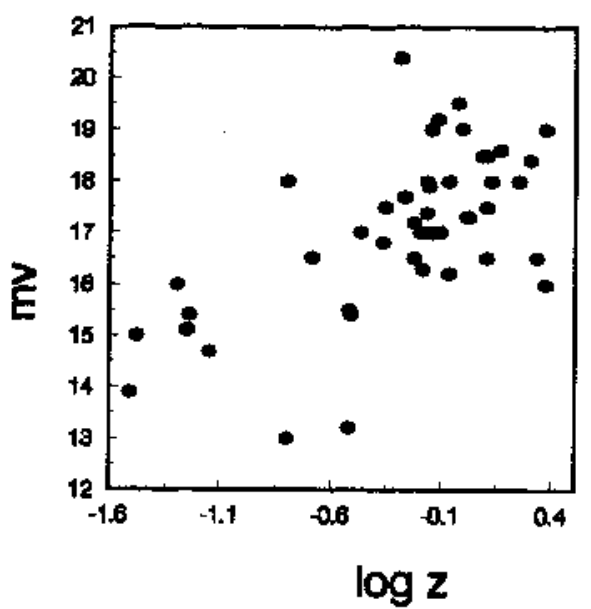

Fig. 2. Relation of visual magnitude $\left(m_{\mathrm{v}}\right)$ and redshift

3.2. Superluminal motion and radio luminosity and redshift

Cruz-Gonzalez \& Carrillo (1991) supposed $\gamma_{\text {jet. }} \approx \beta_{\text {app }}$ and got $\gamma_{\text {jet }} \sim L_{10 \mathrm{GHz}}^{0.28}$ for 30 objects.

From the data in the present paper, we have

$$
\log \beta_{\text {app }}=0.143 \log L_{\mathrm{r}}-4.35
$$

with a correlation coefficient being 0.536 at a confidence level greater than $99 \%$ (see Fig. 7), which is consistent with the Cruz and Carrillo's result. It suggests that the radio emission is strongly enhanced by the Doppler effect. This statistical result also shows that the superluminal motion is associated with the redshift as 


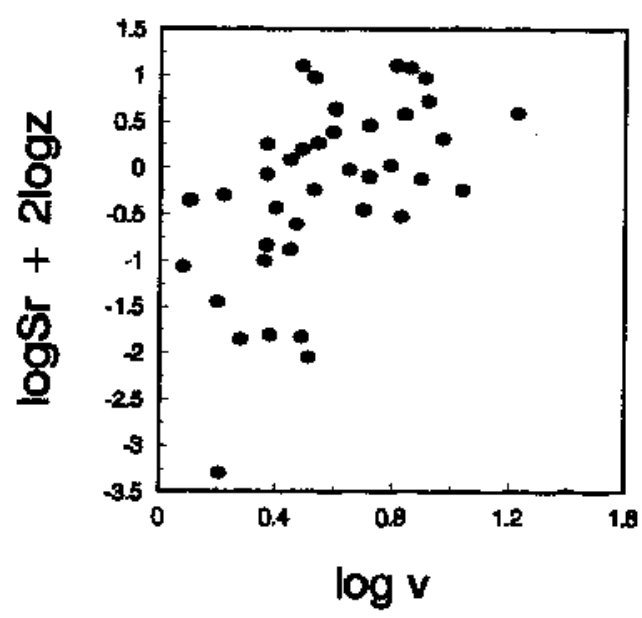

Fig. 3. Relation of $\log S_{\mathrm{r}}+2 \log z$ and superluminal velocity

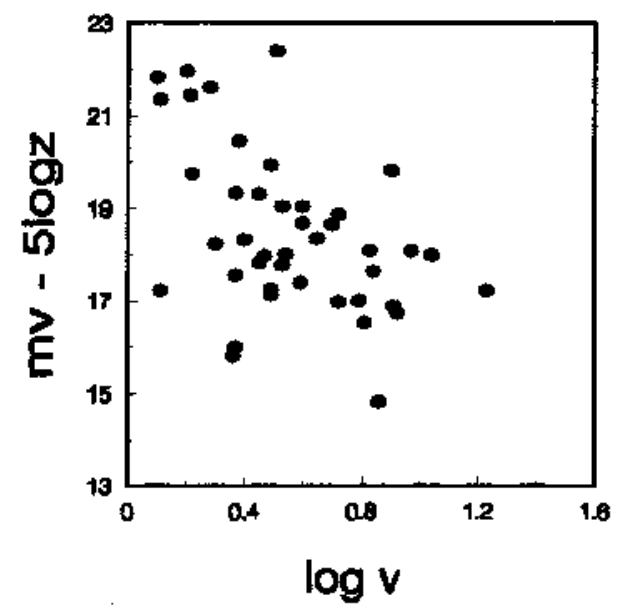

Fig. 4. Relation of $m_{\mathrm{v}}-5 \log z$ and superluminal velocity

$\log \beta_{\text {app }}=(0.22 \pm 0.01) \log z+0.61 \pm 0.001$ with a confidence level greater than $99 \%$ (see Fig. 8): this means that distant objects have a stronger Doppler effect. This correlation may come from the fact that $\log L_{\mathrm{r}}$ is closely associated with the redshift and $\log L_{\mathrm{r}}$ is correlated with the superluminal velocity. This can be confirmed if we use Padovani's method (Padovani 1992) to deal with the relevant data.

If $\gamma_{i j}$ is the correlation coefficient between $x_{i}$ and $x_{j}$, in the case of three variables the correlation between two of them, excluding the effect of the third one, is (Padovani 1992)

$$
\gamma_{12,3}=\frac{\gamma_{12}-\gamma_{13} \gamma_{23}}{\left(1-\gamma_{13}^{2}\right)^{1 / 2}\left(1-\gamma_{23}^{2}\right)^{1 / 2}}
$$

From the data we find that the correlation coefficient between superluminal velocity and redshift, $\gamma_{\mathrm{v}_{z}, L_{\mathrm{r}}}$, excluding the effect of radio luminosity, $L_{\mathrm{r}}$, is 0.132 . The result means there is nearly no correlation between superluminal velocity and redshift: the obtained correlation is only from

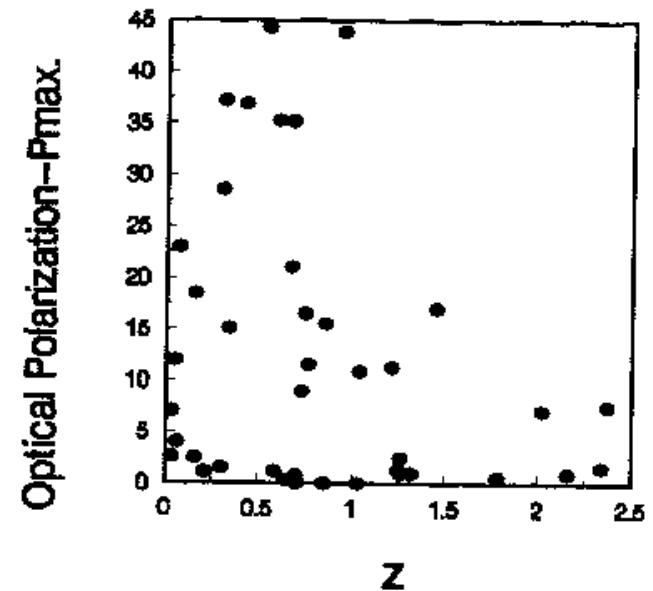

Fig. 5. Relation of optical polarization and redshift

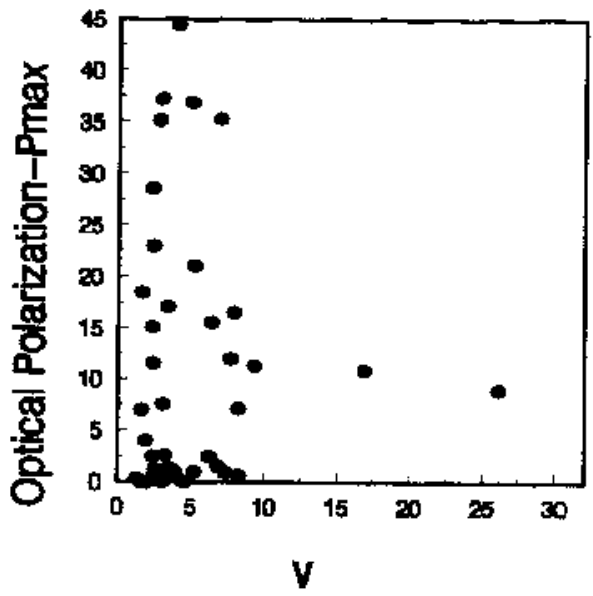

Fig. 6. Relation of optical polarization and superluminal velocity

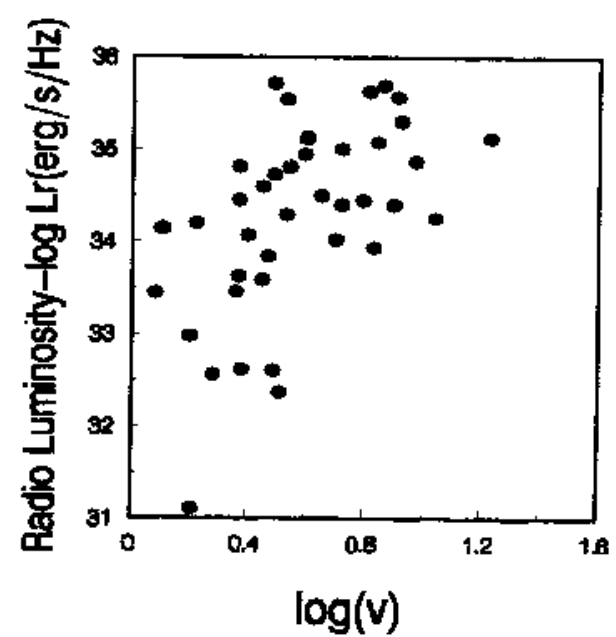

Fig. 7. Relation of radio flux and superluminal velocity 


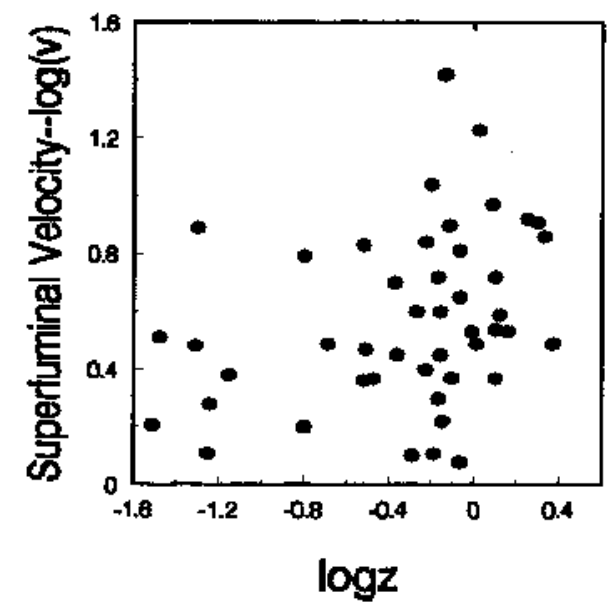

Fig. 8. Relation of superluminal velocity and redshift

an effect of the correlation (at level greater than 99\%) between radio luminosity and redshift and superluminal velocity.

\subsection{Polarization}

23 of the 48 superluminal objects are highly polarized. Figure 5 shows that objects with a lower redshift have a higher polarization than those with higher redshifts, and Fig. 6 shows that objects with lower superluminal velocity have higher polarizations than those with higher velocity. That is why BL Lac objects have higher polarization and lower superluminal velocity than quasars. Figure 9 shows that objects with higher radio luminosity have lower polarization. Figure 10 shows that a higher polarization is associated with a high ratio of radio to optical luminosity$\log L_{\mathrm{r}} / L_{0}$, in agreement with Impey's result (Impey 1987). Figure 11 confirms Wills' result that polarization is associated with the core dominance (Wills 1989).

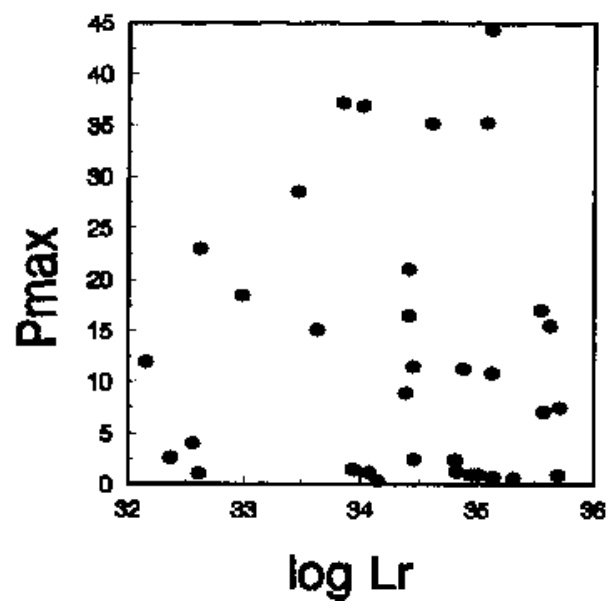

Fig. 9. Relation of optical polarization and radio flux

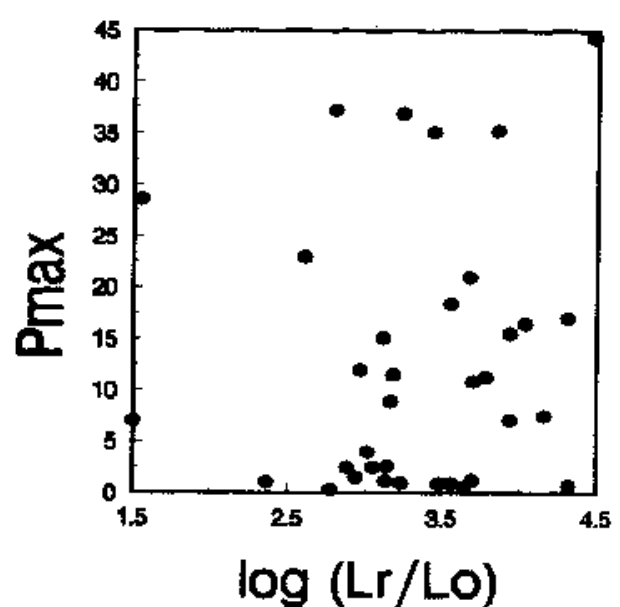

Fig. 10. Relation of polarization and ratio of radio to optical flux

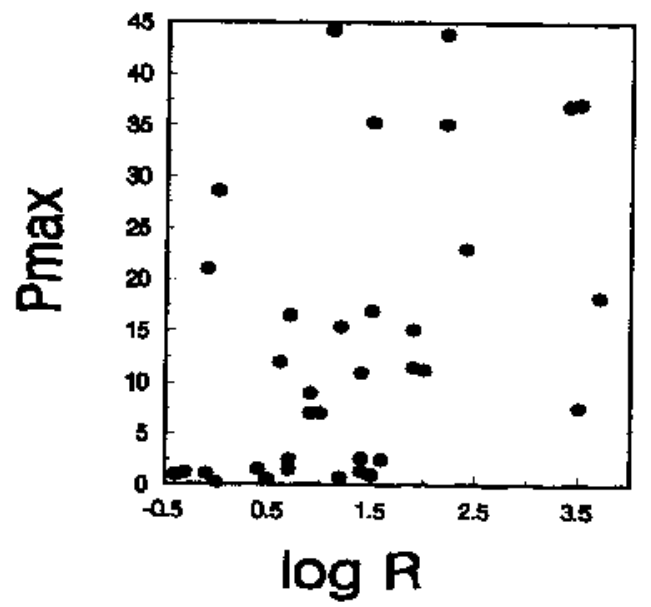

Fig. 11. Relation of optical polarization and core dominance parameter

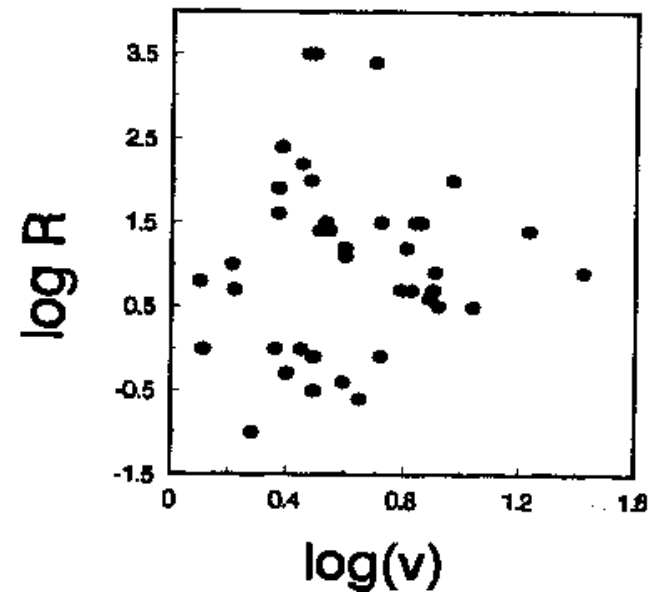

Fig. 12. Relation of superluminal velocity and core dominance parameter 
Table 1. Objects with superluminal motions

Table 1. Objects with superluminal motions

\begin{tabular}{|c|c|c|c|c|c|c|}
\hline $\begin{array}{l}\text { Name } \\
\text { (1) }\end{array}$ & (Q) & $\underset{(9)}{\mathbf{m}}$ & $\begin{array}{l}\beta=\infty / c \\
(d)\end{array}$ & $(5)$ & $\begin{array}{l}\text { Panax } \\
\text { (B) }\end{array}$ & $\begin{array}{l}\text { Refe. } \\
(7)\end{array}$ \\
\hline $0016 \div 791$ & 1.781 & 18.0 & 8.3 & 1,65 & 0.6 & C93,893 \\
\hline $0706+013$ & 2.017 & 38.39 & 8.2 & $2.2 \mathrm{2}$ & 7.1 & $\operatorname{Im} 89, \mathrm{~V} 91, \mathrm{Sg3}$ \\
\hline $0159+744$ & 2.04 & 19.0 & 1.9 & 1,52 & 1,6 & WBT, W92,999 \\
\hline $0212+735$ & 2.367 & 19.0 & $\begin{array}{l}9.9 \\
2.2\end{array}$ & 2,2 & 7.6 & $\begin{array}{l}\text { Ypg2, YQ87, S93, } \\
\text { Seg9 }\end{array}$ \\
\hline $0074+285$ & 1.41 & 18.5 & 9.3 & 1.44 & 1.1 .9 & 593,1094 \\
\hline $0235+164$ & 0.94 & 19.5 & 30. & 2, 85 & 43.9 & W92,F93,Im87,S93 \\
\hline $0913+5921$ & 1.26 & 17.5 & $\begin{array}{c}4.8 \\
5.4\end{array}$ & $1.8+$ & 1.0 & $\begin{array}{l}\text { W92,PB7, Wu92,ImB7 } \\
B 67\end{array}$ \\
\hline $0475+979$ & 0.649 & & $\mathbf{3 . 0}$ & & & PB7, Wug2 \\
\hline $0490+052$ & 0.098 & 15.0 & $\begin{array}{l}2.15 \\
4.9 \\
5.8 \\
4 . J \\
2.1\end{array}$ & 8.44 & 2.6 & $\begin{array}{c}\text { Pa87,K87, S87,S93 } \\
\text { H87,P87,Im67 }\end{array}$ \\
\hline $0454+944$ & 0.16 & 19.0 & 1.6 & 1.99 & 18.4 & P87, G93,E89,593 \\
\hline $0815+820$ & 0.71 & 19.0 & $\begin{array}{c}1,1 \\
2.2\end{array}$ & 1.0 & 0,0 & $\begin{array}{l}\text { We7, W92,S98 } \\
\text { G99 }\end{array}$ \\
\hline $0 \pi 0+450$ & 0.518 & 20.4 & 1.25 & 1,67 & & G99,599 \\
\hline $0716+714$ & 0.3 & 23. 3 & 2,9 & 1,12 & 29.6 & W97,C99, 893 \\
\hline $0724+679$ & 0.846 & 18.0 & $\begin{array}{l}4.8 \\
4.9 \\
4.5\end{array}$ & 1.92 & 0.0 & $\begin{array}{l}\text { W92,W87,699 } \\
\text { S87,HB7 }\end{array}$ \\
\hline $0795+178$ & 0.494 & 16.8 & $\begin{array}{r}2.8 \\
7.2\end{array}$ & 1,99 & 36.9 & $\begin{array}{l}\text { S87,Ma7,PB7,S91,S93 } \\
\text { G93 }\end{array}$ \\
\hline $0836+710$ & 2,16 & 16.5 & $\begin{array}{r}6.2 \\
5.2 \\
10.5\end{array}$ & 2.59 & 1.0 & $\begin{array}{l}\text { WE7,594 } \\
\text { F9s }\end{array}$ \\
\hline $0850+581$ & 1.329 & 18.0 & 9.9 1 & 1.3 & $<1$ & K81,P87,893,工目的 \\
\hline $0851 \div 209$ & 0.300 & 15,4 & $\begin{array}{l}9.18 \\
2.36 \\
3.9\end{array}$ & 9.69 & 37.2 & $\begin{array}{l}\text { S97,S99, Wo9p } \\
\text { FE7 }\end{array}$ \\
\hline $0006+400$ & 0.669 & 18.0 & $\begin{array}{l}6.6 \\
2.4 \\
4.7\end{array}$ & 1.80 & 21.0 & $\begin{array}{l}\text { S93, G93,Pg7 } \\
\text { Fug2 }\end{array}$ \\
\hline $0923+928$ & 0.699 & 17.9 & $\begin{array}{l}9.5 \\
4.0 \\
5.67 \\
2.69\end{array}$ & 8.73 & 0.8 & $\begin{array}{c}\text { Sh87, WgP,Pg7,G93 } \\
\text { Sos3, Fu92 }\end{array}$ \\
\hline $1098+528$ & 0.678 & 17.4 & 2.0 & & & W92,Im87 \\
\hline $1059+81$ & 1.26 & 16.5 & $\begin{array}{l}2.5 \\
2.2\end{array}$ & 1.14 & 2.0 & $\begin{array}{l}\text { WP7, S99 } \\
\text { G99 }\end{array}$ \\
\hline $1040+120$ & 1,09 & 17.9 & 9.1 & 1.47 & 0.0 & P92,PB7,S98 \\
\hline $1101+394$ & 0.031 & 19.9 & $\begin{array}{l}1.96 \\
1.92\end{array}$ & 0.63 & & $\mathrm{Rgg}, \mathrm{Fg4}$ \\
\hline $1187+690$ & 0.852 & 16.9 & 1.37 & 1.00 & B. .9 & W92,PB7, S9d \\
\hline $1150+812$ & 1.25 & 18.5 & $\mathbf{3 . 5}$ & 1.18 & 1.3 & W87, Wu92,599 \\
\hline $\begin{array}{l}175 \theta+295 \\
1222+216\end{array}$ & $\begin{array}{l}0.729 \\
0.435\end{array}$ & $\begin{array}{l}17,0 \\
17+\overline{0}\end{array}$ & $\begin{array}{r}26.1 \\
1.4\end{array}$ & $\begin{array}{l}1.4 \\
0.69\end{array}$ & 9.4 & $\begin{array}{l}\text { F99, Q99, ABO } \\
\text { G93 }\end{array}$ \\
\hline & & & 4.1 & & & P67 \\
\hline
\end{tabular}

*. $\mathrm{S}_{60 \mathrm{O}} \mathrm{GHz}=0.12 \mathrm{Jy}$

\begin{tabular}{|c|c|c|c|c|c|c|}
\hline $\begin{array}{l}\text { Name } \\
\text { (1) }\end{array}$ & ${ }_{(z)}^{Z}$ & (3) & $\begin{array}{l}\beta_{\rightarrow \mathrm{mp}} / \mathrm{C} \\
\text { (4) }\end{array}$ & $\underset{(\overline{5})}{f_{m}}$ & $\begin{array}{l}P_{\text {Max }} \\
\text { (i) }\end{array}$ & $\begin{array}{l}\text { Refs } \\
(7)\end{array}$ \\
\hline $1226+023$ & 0.158 & 19.0 & $\begin{array}{l}5.1 \\
8.0 \\
5.3 \\
6 . \\
6.6\end{array}$ & $42.8 \overline{5}$ & 2.5 & $\begin{array}{c}287, \operatorname{Im} 87,593 \\
587, \mathrm{Bg7}, \mathrm{W} 87\end{array}$ \\
\hline $1259-055$ & 0.538 & $\mathbf{1 7 . 7}$ & $\begin{array}{l}9.0 \\
9.2 \\
2.1 \\
3.1 \\
3.5\end{array}$ & 14.96 & 44.9 & $\begin{array}{l}\text { V91,A60,B87,S98 } \\
\text { S9a,H87, U87 }\end{array}$ \\
\hline $1647+599$ & 0.595 & 16.5 & $\begin{array}{c}9.5 \\
5.9 \\
3.6 \\
8.3\end{array}$ & 10.81 & 35.3 & $\begin{array}{c}\text { A81, W92,B87,S93 } \\
\text { P87,Kris, } 1887\end{array}$ \\
\hline $1642+690$ & 0.761 & 19.2 & 7.9 & 1.39 & 16.5 & K81,P87,S93 \\
\hline $1721+349$ & 0,206 & 16.5 & 3.1 & $0.35^{*}$ & 1.1 & P87, W92, Wu92,Im87 \\
\hline $1749+700$ & 0.77 & 17.0 & 2.35 & $1.4 \overline{0}$ & 11.5 & $\mathbf{5 9 1 , 5 9 3 , G 9 9}$ \\
\hline $1809+784$ & 0.684 & 77,0 & $\begin{array}{l}1.5 \\
9.0 \\
9.9\end{array}$ & 3,64 & 36.2 & $\begin{array}{c}\mathrm{Sg1}, \mathrm{MB5}, \mathrm{Bc} \mathrm{c93} \\
\mathrm{E93,C93}\end{array}$ \\
\hline $1807+698$ & 0,05 & 16,0 & $\begin{array}{r}6.0 \\
7.0 \\
10.0\end{array}$ & 3,26 & 12.0 & S91,L87,S99 \\
\hline $1830+285$ & 0.594 & 17.2 & 2.5 & 1.06 & 1.2 & $899, \mathrm{G99}$ \\
\hline $1845+787$ & 0.057 & 15.4 & 1.9 & 4.98 & 4,0 & K81, $993, P 87, A 80$ \\
\hline $1900+3199$ & 0.635 & 17,0 & $\begin{array}{l}1.4 \\
13.0 \\
10.5 \\
14.0 \\
15.5\end{array}$ & 1.49 & & $\begin{array}{l}\text { P87,5i87, } \\
\text { 887,B87 }\end{array}$ \\
\hline $1929+738$ & 0.\$02 & 15.5 & $\begin{array}{l}9.1 \\
7.0 \\
4.7 \\
5.9 \\
6.7\end{array}$ & 3.94 & 1.5 & $\begin{array}{c}\text { W87,P87,S87 } \\
\text { Wug2,S99 }\end{array}$ \\
\hline $1951+498$ & $0.84 \theta$ & & 1.2 & $0.12^{+}$ & & $7, W u 92$ \\
\hline $1957+405$ & 0.056 & 15.1 & 1.3 & & & Kr99, V91, \\
\hline $2097 \div 777$ & 0.342 & 17.0 & 2.32 & 1.20 & $1 \overline{0} .1$ & $\mathrm{SB1}, \mathrm{K} 90, \mathrm{G99}, \mathrm{S91}, 599$ \\
\hline $2900+420$ & 0.07 & 14.7 & $\begin{array}{l}1.8 \\
2.0 \\
2.4 \\
9.95\end{array}$ & 3.27 & 29.0 & $\begin{array}{l}\mathrm{Sg9}, \mathrm{H87}, \mathrm{Fg3}, \mathrm{SA3} \\
\mathrm{S87}, \mathrm{C93}\end{array}$ \\
\hline 2023-052 & 1.454 & 18.6 & $\mathbf{3 . 8 5}$ & 4,51 & 17.0 & S91,Se8G93,S98 \\
\hline $2990+114$ & 1.097 & 17.3 & $\begin{array}{l}18 . \\
14.2 \\
18.4\end{array}$ & 3.63 & 10.9 & $\begin{array}{c}\text { W92,P67,593 } \\
\text { D94,Im87 }\end{array}$ \\
\hline 2993-148 & 0.976 & 19.0 & $3.3 \overline{0}$ & 0.fil & & V92, V91,G99 \\
\hline $2051+168$ & 0.857 & 16.2 & $\begin{array}{ll}B .8 & 1 \\
9.0 & \\
1.3\end{array}$ & 17,42 & 15.5 & $\begin{array}{c}\mathrm{C77,W92,P87} \text {, } \\
\text { S93,Pa87 }\end{array}$ \\
\hline
\end{tabular}

\section{References to Table 1}

A80: Angel \& Stockman (1980) B87: Brown (1987)

1388: Burbidge \& Ilewitt (1989) C77: Graine (1977)

D94: Dondi \& Ghisellini (1994) 1793: lan ot al. (1993)

F94: Fan at al (1994)

I193: Hotghin ot al. (1993)

Im87: Inpey (1987)

G93: Ghiscllini nt al. (1993)

H87: Hough \& Readhead (1987)

[m88: Empey \& Tapin (1988)

K93: Krichbanm et al. (1993a) K81: Kuhr et al. (1981)

Kr93: Krichbaum et al. (1993b) L87: Lind (1987)

i.a87: Lawrence et al. (1987) M87: Makino et al. (1987)
M85: Holes ot al. (1985) P87: Forcas (1987)

Pa87: Panling-Toth (1987) R93: Reich (1993)

S87: Simon et al. (1987a) S91: Stickel et al. (1991)

S83: Stocke et al. (1983) Sh87: Shalber \& Marscher (1987)

Si87: Simon el al. (1987b) Sc93: Schalinski et al. (1993)

U87: Unwin (1987) V91: Veron \& Veron (1991)

V92: Veron \& Veron (1992) W87: Witzel (1987)

W92: Wills ot al. (1992) Wn92: Wu et al. (1992)

787: Zerngus (1987) 


\subsection{Core dominance parameter}

If the extended emission of radio sources is unbeamed, while the flux of the core is enhanced by beaming, the flux ratio $R$ of the core to the extended component should be a beaming indicator (Scheuer \& Readhead 1979), or $R$ indicates the orientation of the emission (Orr \& Brown 1982). A correlation is then expected between $R$ and the Doppler factor, $\delta$.

Ghisellini et al. (1993) obtained a significant correlation (at the $99.8 \%$ level) between $R$ and $\delta$ and a multiple correlation analysis shows in fact that $R$ is still correlated with $\delta$ at the $98.8 \%$ level even when the effect of the core flux is subtracted, which gives confidence that the core dominance parameter is indeed a beaming indicator.

The measured superluminal velocity and the values of $\delta$ derived from the SSC argument are compared for 46 sources: a significant correlation $(99.7 \%$ level) between the two quantities is obtained and is shown in Fig. 3 (Ghisellini et al. 1993). Obviously, we can expect that the core-dominated objects have a high superluminal velocity. This seems to be true (see Fig. 12). From the data in Table 1, we see that objects with higher redshifts tend to have a larger core dominance parameter, which suggests that distant objects tend to have a beaming effect. This is also consistent with the results that coredominated quasars have a smaller viewing angle with respect to the line of sight $(\phi=8.5 \pm 1.7)$ and a stronger Doppler effect $(\log \delta=0.79 \pm 0.09)$ than BL Lac objects $(\phi=14.0 \pm 2.5, \log \delta=0.49 \pm 0.13)$, lobe-dominated Quasars (LDQ) $(\phi=25.1 \pm 6.4, \log \delta=0.09 \pm 0.26)$ and galaxies $(\phi=40.5 \pm 8.1, \log \delta=0.04 \pm 0.09)$ (Ghisellini et al. 1993).

\section{Conclusions}

From the above analyses, we can come to the following conclusions. Superluminal motion and beaming effect are, to some extent, the same thing: They are related with radio luminosity, redshift, and the core dominance parameter; the optical Doppler factor is related with the superluminal velocity; polarization is associated with redshift, superluminal motion, ratio of radio to optical luminosity, and core dominance parameter; the core dominance parameter is an indicator of the orientation of the emission; probably most of the distant objects are beamed.

Acknowledgements. We thank an anonymous referee for his/her significant suggestions. This work is supported by the National Natural Science Foundation of China and the Natural Science Foundation of Guangdong Province (940557).

\section{References}

Angel J.R.D., Stockman H.S., 1980, ARA\&A 18, 321 Blandford R.D., Konigl A., 1979, ApJ 232, 34
Blandford R.D., Rees M., 1978, in Proc. Pitt. Conf. on BL Lac objects. In: Wolfe A.M. (ed.). Pitt., Univ. of Pitt, 328

Blandford R.D., et al., 1977, Nat 211, 468

Brown I.W., 1987, in Superluminal Radio Sources. In: Zensus A. \& Pearson T.J. (eds.). Cambridge Univ. Press, 129

Burbidge G., Hewitt A., 1989, in BL Lac objects. In: Maraschi L., Maccacaro T. \& Ulrich M.-H. (eds.). Berlin: Springer, 412

Cohen M.H., 1989, in BL Lac objects. In: Maraschi L., Maccacaro T. \& Ulrich M.-H. (eds.). Berlin: Springer, 13

Crain E.R., 1977, A handbook of Quasistellar and BL Lac objects. Pattchart Publishing House, Tucson, Ariz, Vol. 56, 63

Cruz-Gonzales I., Carrillo R., 1991, in Variability of Blazars. In: Valtaoja E. \& Valtonen M. (eds.). Cambridge: Sydny, 256

Dondi L., Ghisellini G., 1995, MNRAS 273, 583

Fan J.H., et al., 1993, ApJ 415, 113

Fan J.H., et al., 1994, A\&AS 105, 415

Ghisellini G., et al., 1993, ApJ 407, 65

Hough D.H., et al., 1993, MPIfR, Preprint Ser. No. 502

Hough D.H., Readhead A.C., 1987, in Superluminal Radio Sources. In: A. Zensus A. \& Pearson T.J. (eds.). Cambridge Univ. Press, 114

Impey C.D., 1987, in Superluminal Radio Sources. In: Zensus A. \& Pearson T.J. (eds.). Cambridge Univ. Press, 233

Impey C.D., Tapia S., 1988, ApJ 333, 666

Krichbaum T.P., et al., 1993a, MPIfR, Preprint Ser. No. 502

Krichbaum T.P., et al., 1993b, MPIfR, Preprint Ser. No. 508

Kuhr H., Schmidt D.A., 1990, AJ 99, 1

Kuhr H., Witzel A., Pauliny-Toth I.I.K. and Nauber U., 1981, A\&AS 45, 367

Lind K.R., 1987, in Superluminal Radio Sources. In: Zensus A. \& Pearson T.J. (eds.). Cambridge Univ. Press, 180

Lawrence C.R., et al., 1987, in Superluminal Radio Sources. In: Zensus A. \& Pearson T.J. (eds.). Cambridge Univ. Press, 260

Madau M., Ghisellin G., Persic M., 1987, MNRAS 224, 257

Makino F., 1987, ApJ 313, 662

Marscher A.P., 1978, ApJ 219, 392

Moles M., et al., 1985, ApJS 58, 255

Orr M.J.L., Brown I.W.A., 1982, MNRAS 200, 1067

Padovani P., 1992, A\&A 256, 399

Porcas R.W., 1987, in Superluminal Radio Sources. In: Zensus A. \& Pearson T.J. (eds.). Cambridge Univ. Press 12

Pauliny-Toth I.I.K., 1987, in Superluminal Radio Sources. In: Zensus A. \& Pearson T.J. (eds.). Cambridge Univ. Press, 55

Reich W., et al., 1993, MPIfR, Preprint Ser. No. 507

Schalinski C., et al., MPIfR, Preprint Ser. No. 502

Scheuer P.A.G., Readhead A.C.S., 1979, Nat 277, 182

Shafber D.B., Marscher A.P., 1987, in Superluminal Radio Sources. In: Zensus A. \& Pearson T.J. (eds.). Cambridge Univ. Press, 67

Simon R.S., et al., 1987a, in Superluminal Radio Sources. In: Zensus A. \& Pearson T.J. (eds.). Cambridge Univ. Press, 155

Simon R.S., et al., 1987b, in Superluminal Radio Sources. In: Zensus A. \& Pearson T.J. (eds.). Cambridge Univ. Press, 72 
Stickel M., et al., 1991, ApJ 374, 431

Stocke J.T., et al., 1983, ApJ 297, 458

Surdej J., et al., 1992, ESO Sci. Prep. No. 898

Unwin S.C., 1987, in Superluminal Radio Sources. In: Zensus A. \& Pearson T.J. (eds.). Cambridge Univ. Press, 34

Veron-Cetty M.-P., Veron P., 1992, Observatoire de Haute Provence, Pre-Publication No. 71

Veron-Cetty M.-P., Veron P., 1991, ESO Sci. Rep. No. 10

Witzel A., 1987, in Superluminal Radio Sources. In: Zensus A. \& Pearson T.J. (eds.). Cambridge Univ. Press, 83
Wills B.J., et al., 1992, ApJ 398, 454

Wills B.J., 1989, in BL Lac objects. In: Maraschi L., Maccacaro T. \& Ulrich M.-H. (eds.). Berlin: Springer, 109

Worrall D.M., 1986, ApJ 303, 589

Wu S.Y., Quirrenbach Witzel A., 1992, Chinese Sci. Ser. A 7, 743

Xie G.Z., et al., 1993, A\&A 278, 6

Zensus J.A., 1987, in Superluminal Radio Sources. In: Zensus A. \& Pearson T.J. (eds.). Cambridge Univ. Press, 26 